\title{
An obstructive role of NK cells on metastatic growth of clear-cell sarcoma cells in a xenoplant murine model
}

\author{
YUKI HANAMATSU, CHIEMI SAIGO, YUSUKE KITO and TAMOTSU TAKEUCHI
}

Department of Pathology and Translational Research, Gifu University Graduate School of Medicine, Gifu 501-1193, Japan

Received December 27, 2019; Accepted June 11, 2020

DOI: $10.3892 / \mathrm{mco} .2020 .2171$

\begin{abstract}
Clear cell sarcoma (CCS) affects the deep soft tissues in young adults and is known to have high rates of metastasis, including lymphatic metastasis. In our previous study an xenoplant model of CCS was established, which exhibited local tumor growth, lymphatic metastasis, and distant metastasis in SCID-Beige mice. In the current study, the role of NK cells during metastasis in the same xenoplant murine model was investigated. Injection of murine or human NK cells significantly suppressed the metastasis of HS-MM CCS cells in SCID-Beige mice. Notably, reverse transcription-quantitative PCR analysis demonstrated that injection of NK cells did not alter the mRNA expression levels of ERSR1-ATF1, which is specifically transcribed in CCS, in the buffy coat of circulating blood cells of HS-MM-xenoplanted SCID-Beige mice. BALB/c nude mice xenoplanted with HS-MM cells exhibited local growth without evident metastasis, whereas inoculation with the anti-asialo-GM1 antibody, which has previously been found to abolish NK-cell activity, resulted in metastasis of HS-MM cells in BALB/c nude mice. The injection of the anti-CD96 antibody, which increases the cytotoxicity of NK cells, significantly suppressed the metastasis of HS-MM cells in SCID-Beige mice. These results indicated that NK cells impaired the metastatic tumor microenvironments in the present mice xenoplant model.
\end{abstract}

\section{Introduction}

Clear cell sarcoma (CCS) is characterized by a chromosomal $\mathrm{t}(12 ; 22)$ (q13; q12) translocation resulting in a fusion between the Ewing sarcoma (EWSRl) and activating transcription factor 1 (ATF1) genes (1). Detection of the EWSR1-ATF1 chimera gene product is widely used as a highly sensitive diagnostic test for CCS (2). CCS affects the deep soft

Correspondence to: Professor Tamotsu Takeuchi, Department of Pathology and Translational Research, Gifu University Graduate School of Medicine, Yanagido 1-1, Gifu 501-1193, Japan

E-mail: takeutit08@gmail.com

Key words: metastatic tumor microenvironment, clear cell sarcoma, metastatic model, NK cells, ERSRI-ATF1 tissues predominantly in young adults, aged between 15 and 35 years of age and is known to have high rates of metastasis, in worldwide $(3,4)$. For example, Chung and Enzinger (4) reported that 50 out of 115 patients had died from metastatic tumors in 1983. Despite progress in the different treatments available, the prognosis of patients with CCS is still poor, as $30 \%$ of patients have metastases at the time of diagnosis (5). Lymphatic metastasis is rare in other types of malignant soft tissue tumors; however, is commonly detected in CCS (4,6). A previous study reported that positive sentinel nodes were identified in 2 out of 42 patients with synovial sarcoma, compared with 6 out of 12 patients with CCS $(6,7)$. Radical surgical resection is the first line of treatment for CCS; however, the rate of local recurrence can be as high as $84 \%$ and the rate of late metastases (10 years following surgery) can be up to $63 \%$, which have been associated with a 5 to 20 -year survival rate of $67-10 \%$ (8). As CCS has been found to be resistant to conventional soft tissue sarcoma chemotherapy regimens, for example doxorubicin-based chemotherapy (8), therefore therapies that specifically control metastasis are urgently required.

For the development of novel targeted therapies, a CCS model, that exhibited similar clinicopathological features, including metastatic potential, was established in our previous study, by xenoplanting HS-MM CCS cells into SCID-Beige mice (9), which was subsequently used to investigate the pharmacological effect of a lipoate analogue, CPI-613 (9) or an anti-CD151 antibody (10).

During the establishment of the CCS murine model, in both SCID-Beige and BALB/c nude mice, it was found that the latter mice showed no metastasis (9). A SCID-Beige mouse is a double-mutant mouse strain, with impaired lymphoid development and weak NK cell activity (11). By contrast, the BALB/c nude mouse has robust NK cell activity, which complements deficiencies in thymus-dependent $\mathrm{T}$ lymphocyte function (12).

The aim of the present study was to investigate whether NK cells impaired metastasis of CCS in murine xenoplanted models.

\section{Materials and methods}

HS-MM clear cell sarcoma cell line. The HS-MM CCS cell line was previously established and characterized in our laboratory $(13,14)$. HS-MM cells harbor a canonical genetic background with $\mathrm{t}(12 ; 22)$ (q13; q12) of CCS, which results in an EWSR1-ATF1 fusion gene (1). Cells were cultured in 
Dulbecco's modified Eagle's medium (DMEM; Gibco; Thermo Fisher Scientific, Inc.) containing 10\% heat-inactivated fetal bovine serum (HyClone; GE Healthcare Life Sciences). Cells were passaged for no more than six months following resuscitation. Cells were screened periodically for mycoplasma contamination using DAPI staining.

Mice. The animal experiments were conducted at Gifu University under the guidelines for animal experimentation and followed the Japanese Law for the Humane Treatment and Management of Animals. The experimental protocol was approved by the Animal Care Committee of Gifu Graduate School of Gifu (approval no. 27-80 and 2020-066). A total of $3 \mathrm{H}-2 \mathrm{~d}$ congenic strains, namely, SCID-Beige (CB17.Cg-Prkdc ${ }^{\text {scid }}$ Lyst $^{\text {tog-J }} / \mathrm{Crl}^{\text {Crlj; }}$; weight, $18.3-21.1 \mathrm{~g} ; \mathrm{n}=19$ ), BALB/c nude (BALB/c-nu; CAnN.Cg-Foxn $1^{\text {nu }} / \mathrm{Crl}^{\mathrm{Crlj}}$; weight 17.0-18.9 g; $\mathrm{n}=10$ ), and $\mathrm{BALB} / \mathrm{c}$ mice (weight 19.7-22.4 g; $\mathrm{n}=5$ ), were purchased from Charles River Laboratories, Inc. All mice were female and 8-weeks-old, and kept under specific pathogen-free conditions in isolated and ventilated cages, with free access to food and water, and maintained with a 12 -h light/dark cycle at $23^{\circ} \mathrm{C}$. Every effort was made to minimize suffering as previously described $(9,10)$. Briefly, murine behavior and body weight were monitored twice per week. A $50 \mathrm{mg} / \mathrm{ml}$ solution of pentobarbital in sterile saline was administered intraperitoneally to SCID-Beige and $\mathrm{BALB} / \mathrm{c}$ nude mice, at a dose of $120 \mathrm{mg} / \mathrm{kg}$ for euthanasia and were confirmed to have died when the heart stopped beating. Time to initiation of tissue collection was $15 \mathrm{~min}$. To obtain the spleen from the BALB/c mice, carbon dioxide inhalation was used, as the method of euthanasia until the mice stopped breathing. Carbon dioxide (100\%) was introduced into a 5.7L cage, initially containing normal air conditions, with the lid closed, at a low-flow rate of $30 \%$ volume displacement per minute. Before the experiments, humane endpoints were set as follows: i) When the body weight following tumor cell inoculation decreased by $>10 \%$ compared with the initial weight of the mouse; ii) when the behavioral observations, such as food/water consumption and daily activity, monitored during the experiment, were reduced by $50 \%$; and iii) when the fur became dull. Finally, if ulceration was observed at the transplantation site, the mouse was sacrificed.

However, among the 19 SCID-Beige and $10 \mathrm{BALB} / \mathrm{c}$ $\mathrm{nu} / \mathrm{nu}$ mice examined, there was no suffering in any of the mice using the aforementioned criteria nor were any of the mice found dead. In addition, $5 \mathrm{BALB} / \mathrm{c}$ mice, which were used to obtain the NK-cells exhibited no suffering prior to euthanasia.

Xenoplantation and injection of NK cell fraction. Xenoplantation of HS-MM cells was performed as previously described $(9,10)$. Briefly, SCID-Beige mice were injected with $2.5 \times 10^{7} \mathrm{HS}-\mathrm{MM}$ cells into the aponeuroses of the thighs, subsequently 9 mice were randomly divided to three groups, of three mice. A total of $0.3 \mathrm{ml}$ saline, with or without $1 \times 10^{4}$ murine or $1.5 \times 10^{6}$ human NK cells was intravenously injected to SCID-Beige mice two weeks following HS-MM xenoplantation. A total of 8 weeks following xenoplantation, the mice were sacrificed, and the tumor size was measured using a caliper and the following formula: Volume $=\left(\right.$ width $^{2} \mathrm{x}$ length $) / 2$.
NK cells were harvested from splenic cells of BALB/c mice using a mouse NK cell isolation kit (Stemcell Technologies, Inc.), or collected from peripheral blood samples from a healthy volunteer using negative selection and a NK Cell Isolation kit, MACS system (Miltenyi Biotec, Inc.) according to the manufacturer's protocol. Written informed consent was provided from the healthy volunteer prior to the start of the study. The study was approved by the Committee of Gifu Graduate School of Gifu, Japan (approval no. 27-80, 2020-066).

The isolated human NK cells were cultured in T25 flasks (Thermo Fisher Scientific, Inc.) with $10 \mathrm{ml}$ KBM502 medium (Kohjin-bio, Co., Ltd.) at $37^{\circ} \mathrm{C}$ in a humidified incubator with $5 \% \mathrm{CO}_{2}$ for seven days.

Reverse transcription-quantitative PCR (RT-qPCR). Total RNA was extracted using the Qiagen RNeasy Kit (Qiagen $\mathrm{GmbH}$ ). cDNA synthesis from total RNA and subsequent PCR was performed using the RT-PCR kit (Takara Bio Inc.) according to the manufacturer's instructions and as previously described (15). qPCR was performed using the FastStart Essential DNA SYBR ${ }^{\circledR}$ Green master mix according to the manufacturer's instructions using a LightCycler (both Roche Diagnostics $\mathrm{GmbH}$ ) as previously described (16). Briefly, cells were isolated from the buffy coat of $0.5 \mathrm{ml}$ right ventricular blood, obtained from SCID-Beige mice injected with murine NK cells $(n=3)$ and the mice without NK cell injection group $(n=3)$ after euthanasia. Total RNA was extracted and reverse transcribed, following which cDNA ( $1 \mu 1$ per reaction) was diluted with PCR mix, containing 0.2 pmol primers, to a final reaction volume of $20 \mu \mathrm{l}$. The following qPCR primers were used: EWSR1-ATF1 forward, 5'-CATGAGCAGAGG TGGGCG-3' and reverse, 5'-CCCCGTGTATCTTCAGAA GATAAGTC-3'; and GAPDH forward, 5'-GAAATCCCA TCACCATCTTCCAGG-3' and reverse, 5'-GAGCCCCAG CCTTCTCCATG-3'. Samples were performed in triplicate, and expression levels of each target gene were analyzed with a LightCycler system using the $2^{-\Delta \mathrm{CC}}$ method described by Livak and Schmittgen (17). For each triplicate set, the $\Delta \mathrm{Cq}$ values were normalized to GAPDH expression in the control group. The values of the target group were then calculated as the fold change relative to the mean values of the control group (control; set to 1.0). An unpaired Student's t-test was used to determine significant differences in gene expression among the treatment groups. $\mathrm{P}<0.05$ was considered to indicate a statistically significant difference.

Anti-asialo GM1 antibody treatment. A rabbit antibody targeting asialo-GM1 was purchased from FUJIFILM Wako Pure Chemical Corporation (cat no. 014-09801). BALB/c nude mice were intraperitoneally injected with or without $300 \mu \mathrm{g}$ anti asialo-GM1 antibody at 7, 14, 21, and 28 days following xenoplantation with $2.5 \times 10^{7} \mathrm{HS}-\mathrm{MM}$ cells.

Anti-CD96 antibody treatment. Rat monoclonal antibody against murine CD96 (clone 630612) was purchased from R\&D Systems Inc. SCID-Beige mice were intraperitoneally injected with or without $12.5 \mu \mathrm{g}$ anti-CD96 antibody at 7, 14, 21, and 28 days following injection with HS-MM cells. Intraperitoneal metastatic lymph nodes were dissected using a stereomicroscope. Total metastatic lymph nodes were then weighed. 
A

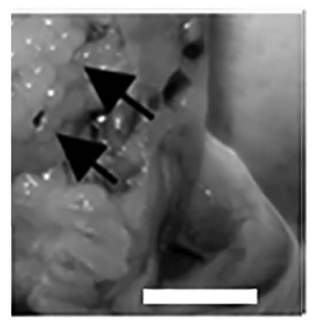

B

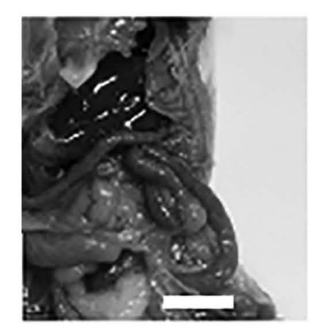

C

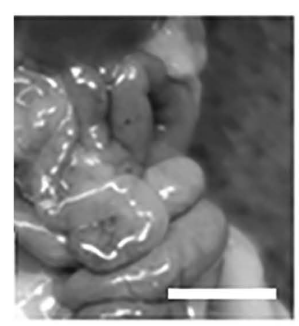

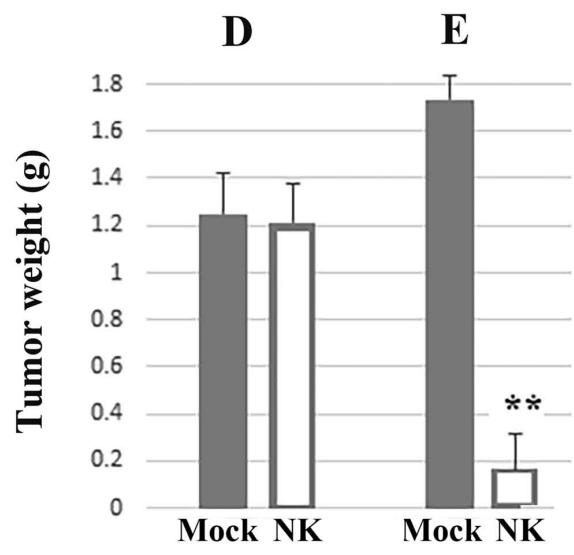

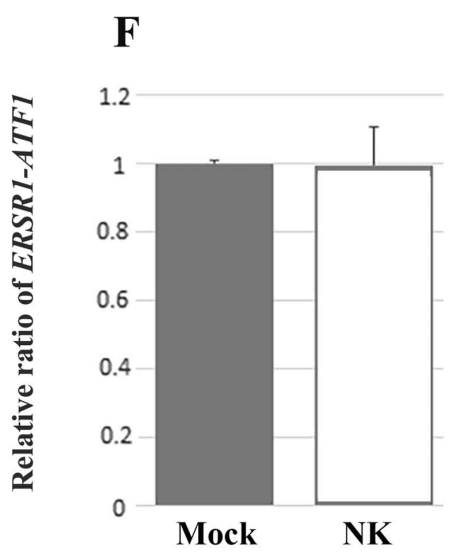

G

H
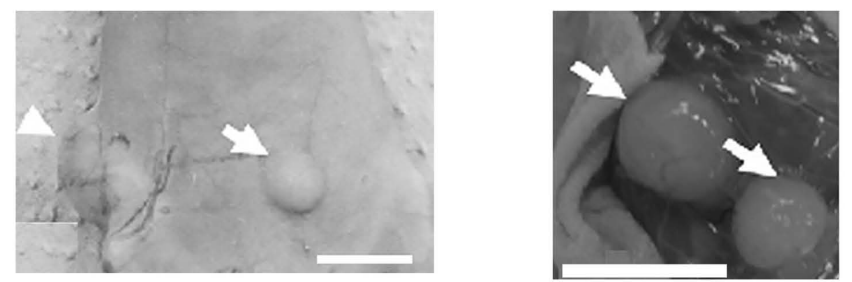

Figure 1. NK cells play a crucial role in suppressing metastasis of xenoplanted HS-MM cells. (A) SCID-Beige mice showed prevalent mesenteric lymph node metastasis (indicated by the arrows), whereas SCID-Beige mice transplanted with HS-MM cells and (B) murine and (C) human NK cells showed marked reduction of metastasis from the few macroscopic metastatic tumors found. (D) The tumor weight at the injection sites and (E) the total weight of the collected, disseminated mesenteric tumors, 10 days following the injection of human NK cells. The growth of xenotransplanted HS-MM cells at the injection sites was not altered by the transfer of human NK cells, whereas the growth of multiple disseminated tumors in the mesentery was inhibited. Data are expressed as the mean $\pm \mathrm{SD}(\mathrm{n}=3)$. Student's t-test was used. ${ }^{* *} \mathrm{P}<0.01$ vs. mock. (F) Reverse transcription-quantitative PCR showed similar expression of ERSR1-ATF1 chimera gene transcripts from blood buffy coat cells obtained from SCID-Beige mice injected with murine NK cells (n=3) and the mock group $(\mathrm{n}=3)$. (G) Anti-asialo-GM1 antibody treatment promoted distal metastasis (indicated by arrow) in BALB/c nude mice injected with HS-MM cells (arrowhead indicates the transplanted tumor). (H) Lymphatic metastasis was also observed in the BALB/c nude mice treated with anti-asialo-GM1 antibody (arrow). Scale bars, $1 \mathrm{~cm}$. NK, natural killer; Mock, non-NK cell transplanted mice; EWSR1, EWS RNA binding protein 1; ATF1, activating transcription factor 1.

Histopathological analysis. The tumor tissue sections from the primary injection site and metastatic tumor in mice, which were transplanted with murine NK cells, treated with anti-CD96 antibody, and control mice were fixed for $48 \mathrm{~h}$ at room temperature with $10 \%$ neutral buffered formalin, embedded in paraffin, and cut into $4-\mu \mathrm{m}$ thick sections. Paraffin slides were deparaffinized in xylene, twice, for $10 \mathrm{~min}$ and dehydrated in a descending alcohol series $(95,90,70 \%)$ for 2 min, each time. Following rinsing with water, tissue sections were stained with Mayer hematoxylin solution for $8 \mathrm{~min}$, then with eosin Y solution for $1 \mathrm{~min}$, both at room temperature. The tissue sections were evaluated using light microscopy at x400 magnification.

Statistical analysis. The results are presented as the mean \pm standard deviation of triplicate experiments. Statistical analyses were performed using EZR (v1.27) (18), which is a graphical user interface for R (19). Statistical significance was determined using an unpaired Student's t-test, Welch's t-test or one-way ANOVA with the Bonferroni correction. $\mathrm{P}<0.05$ was considered to indicate a statistically significant difference.

\section{Results}

NK cells abrogate metastasis of CSS without affecting the growth of tumors at the primary injection site in SCID-Beige xenoplant model. The HS-MM cells were successfully transplanted into the soft tissues of the thighs of all the SCID-Beige mice. At the end of the experiments, the body weights of the mice in each group were as follows: $22.5,21.8$, and $22.9 \mathrm{~g}$ for the control mice; 20.8, 21.0, and $22.2 \mathrm{~g}$ for the murine NK cell-transplanted mice; and 20.7, 20.6, and $21.2 \mathrm{~g}$ for the human NK cell-transplanted mice; the weights of the control mice were significantly higher compared with those of 
A

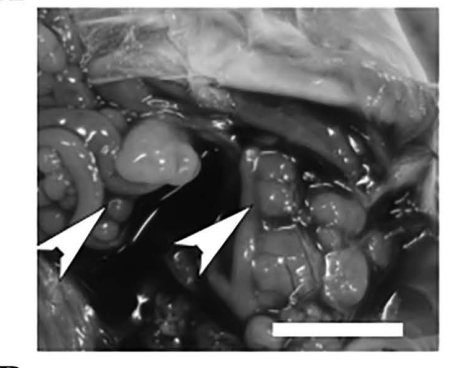

B

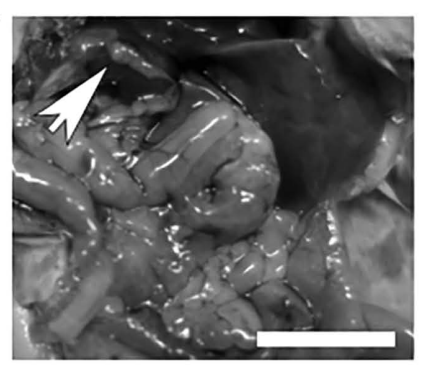

C

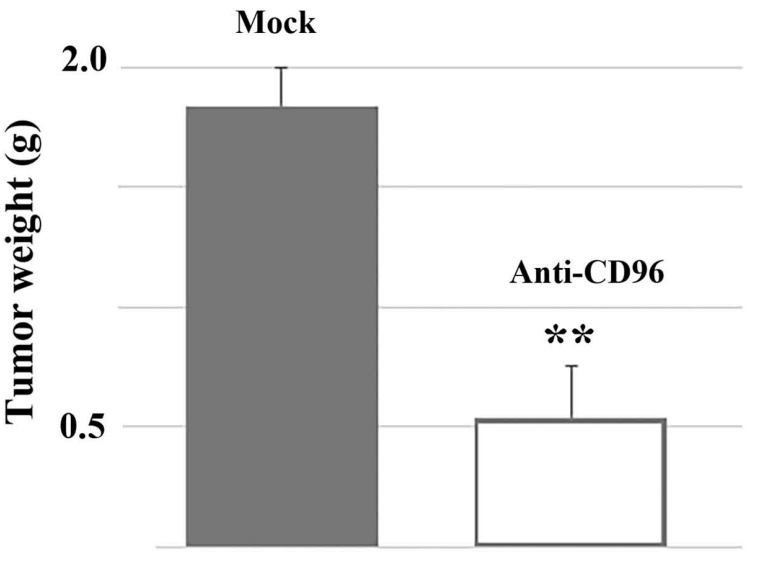

Figure 2. Anti-CD96 antibody treatment blocked metastasis of HS-MM cells in SCID-Beige mice. The metastasis of HM-MM cells in (A) control and (B) SCID-Beige mice injected with HS-MM cells and anti-CD96 antibody, to increase the lytic activity of NK cells. The arrow-head indicates the tumors. (C) Total weight of collected, disseminated mesenteric tumors 10 days following the injection of human NK cells. Data are expressed as mean \pm SD ( $=5$ ). Student's t-tests was performed. ${ }^{* *} \mathrm{P}<0.01$. Scale bar, $1 \mathrm{~cm}$. NK, natural killer.

the human NK cell-transplanted mice ( $\mathrm{P}=0.039$; Fig. $\mathrm{S} 1)$. The metastasis of HS-MM was examined using a stereomicroscope following euthanasia. Histopathological examination of the primary injection site, with or without murine NK cell-transplantation or anti-CD96 antibody-injection in the tumor tissues was also performed. There were no histopathological alternations, such as the presence of necrosis or lymphocyte infiltration at the primary injection site by these treatments (Fig. S2). Therefore, the results of the present study suggest that the activity of NK cells might abrogate the formation of the metastatic tumor but does not affect the primary tumor.

Lymph node and distant metastasis, i.e., lung metastasis, were detected at 8 weeks, following injection in all the inoculated mice. Representative images of mesenteric lymph node metastasis are shown in Fig. 1A. By contrast, murine or human NK cell transplantation inhibited metastasis of HS-MM cells in SCID-Beige mice (Fig. 1B and C, respectively). The growth of xenoplanted HS-MM cells at the injection sites was not altered by the adoptive transfer of human NK cells $(1.25 \pm 0.18$ vs. $1.21 \pm 0.17 \mathrm{~g}$ for non-NK cell transplanted and NK cell-transplanted group, respectively; $n=3 ; P=0.800$; Fig. 1D), whereas the growth of multiple disseminated tumors in the mesentery was significantly inhibited $(1.74 \pm 0.10 \mathrm{vs} .0 .17 \pm 0.15 \mathrm{~g}$ in the non-NK cell transplanted and NK cell-transplanted group, respectively; $\mathrm{n}=3$; $\mathrm{P}<0.001$; Fig. 1E).

$N K$ cells did not affect the mRNA expression of EWSRI-ATF1 in circulating blood cells. NK cell transplantation did not induce significant changes in the transcriptional expression of EWSR1-ATF1 in the buffy coat cells from the right ventricular blood of SCID-Beige mice (1 vs. 0.996 \pm 0.11 in the control and murine NK cell-transplanted group; $\mathrm{n}=5 ; \mathrm{P}=0.900$; Fig. 1F).

Effect of anti-asialo GM1 and anti-CD96 antibody on metastasis of CCS. At the end of the experiments, the body weights of the mice were as follows: 20.9, 21.1,21.2, 20.7, and $21.4 \mathrm{~g}$ for the control mice and 21.2, 21.0, 21.0, 21.7, and $22.3 \mathrm{~g}$ for the anti-asialo-GM1 antibody-treated mice. No significant differences were observed in the body weights of the control and anti-asialo-GM1 antibody-treated mice $(\mathrm{P}=0.259)$. Notably, treatment with the anti-asialo GM1 antibody markedly promoted the metastasis of xenoplanted HS-MM cells in the BALB/c nude mice (Fig. $1 \mathrm{G}$ and $\mathrm{H}$ ).

Previous studies have found CD96 to be a negative regulator of the NK-cell response in the tumor microenvironment by using different tumor models $(20,21)$. Therefore, it was investigated whether forced activation of residual NK cells could reverse the metastasis of HS-MM cells in SCID-Beige mice. The representative data are shown in Fig. 2. Notably, intraperitoneal injection of anti-CD96 antibody significantly reversed the metastasis of HS-MM cells in the present SCID-Beige mice model $(1.84 \pm 0.16$ vs. $0.54 \pm 0.21 \mathrm{~g}$ in the control and NK cell-transplanted group, respectively; $n=5$; $\mathrm{P}<0.001)$. Furthermore, at the end of the experiments, the body weights of the mice were as follows: $21.8,21.0,20.9,21.4$, and $21.6 \mathrm{~g}$ for the control mice and 21.4, 21.8, 21.3, 21.0, and $22.3 \mathrm{~g}$ for the anti-CD96 antibody-treated mice. No significant differences were observed in the body weights of the two groups $(\mathrm{P}=0.460)$.

These results demonstrated the critical role of NK cell activity in suppressing metastasis of xenoplanted HS-MM cells.

\section{Discussion}

The rapid growth and robust metastatic activity of the HS-MM cells was observed during the short duration of the experiments, which was 8 weeks following transplantation. Therefore, the present murine model, which caused distant metastasis, as observed in humans, was a suitable metastatic tumor model as previously described $(9,10)$. 
Metastasis is a complex process, which proceeds in several steps, including local invasion and intravasation, transport through the circulation, extravasation, micrometastasis, and subsequent development of overt metastatic tumors (22). In the present study, CCS-specific transcription of ERSR1-ATF1 chimera gene was equally detected in the buffy coats in cells from xenoplanted SCID-Beige mice and in mice transplanted with murine NK cells (Fig. 1F). This result indicated the expression of the EWSRI-ATF1 fusion gene product in the blood was not reduced by adoptive NK cell transplantation in the present murine model. We hypothesize that NK cells suppress the formation of the tumor environment during the micrometastasis step, including foreign tumor microenvironments in distant tissues. This is consistent with the recent hypothesis that NK cells are recruited by a subpopulation of patrolling non-classical monocytes to the extravasating tumor cells to prevent the development of a metastatic microenvironment (23).

CD96 has recently been found to be a negative regulator of NK cell activity (18). The findings from the present study also revealed that anti-CD96 antibody treatment markedly reduced metastasis of HS-MM cells in SCID-Beige mice, in which NK cell activity against the YAC cell line was $\sim 50 \%$ lower compared with that in SCID mice (11). Thus, inoculation with the anti-CD96 antibody, which also enhances NK cell activity (23), or targeting other inhibitory immune checkpoint receptors for $\mathrm{NK}$ cell, for example $\mathrm{T}$ cell immunoglobulin and immunoreceptor tyrosine-based inhibitory motif domain (24), could potentially be used for regulating tumor metastasis through the inhibition of micrometastasis in the tumor environment to improve survival in patients with CCS following surgery. In the present study, adoptively transferred human NK cells significantly suppressed the metastasis of human CCS cells. This suggests that adoptive transfer of NK cells in patients with CCS could have a potential therapeutic effect. The present study also demonstrated that anti-asialo-GM1 antibody, which is well-known to impair the NK cell activity (25), induced metastasis of CCS in BALB/c nude mice. Therefore, it should be further examined whether surgical treatment might impair NK cell activity and attribute to the metastasis of CCS. Furthermore, extensive studies are also required to unravel whether NK cells directly attach to the CCS at the micrometastasis site or whether NK cells are important to inhibit the formation of micrometastasis environment.

In conclusion, the results of the present study indicated that NK cells may impair the metastatic tumor microenvironment in the mouse xenoplant model.

\section{Acknowledgements}

Not applicable.

\section{Funding}

No funding was received.

\section{Availability of data and materials}

All data generated or analyzed during this study are included in this published article.

\section{Authors' contributions}

TT conceived and designed the study. YH and TT analyzed, interpreted data and drafted the manuscript. YH, CS, and YK performed the experiments. All authors participated in the writing of the manuscript and have read and approved the final manuscript.

\section{Ethics approval and consent to participate}

The animal experiments were approved by the Animal Care Committee of Gifu Graduate School of Gifu, Japan (approval no. 27-80). The use of human tissues was approved by the Committee of Gifu Graduate School of Gifu, Japan (approval no. 27-80, 2020-066). Written informed consent was provided by the healthy volunteer.

\section{Patient consent for publication}

Not applicable.

\section{Competing interests}

The authors declare that they have no competing interests.

\section{References}

1. Zucman J, Delattre O, Desmaze C, Epstein AL, Stenman G, Speleman F, Fletchers CD, Aurias A and Thomas G: EWS and ATF-1 gene fusion induced by $\mathrm{t}(12 ; 22)$ translocation in malignant melanoma of soft parts. Nat Genet 4: 341-345, 1993.

2. Thway $\mathrm{K}$ and Fisher C: Tumors with EWSR1-CREB1 and EWSR1-ATF1 fusions: The current status. Am J Surg Pathol 36: e1-e11, 2012.

3. Enzinger FM: Clear-cell sarcoma of tendons and aponeuroses. An analysis of 21 cases. Cancer 18: 1163-1174, 1965.

4. Chung EB and Enzinger FM: Malignant melanoma of soft parts. A reassessment of clear cell sarcoma. Am J Surg Pathol 7: 405-413, 1983.

5. Ibrahim RM, Steenstrup Jensen S and Juel J: Clear cell sarcoma-A review. J Orthop 15: 963-966, 2018.

6. Andreou D and Tunn PU: Sentinel node biopsy in soft tissue sarcoma. Recent Results Cancer Res 179: 25-36, 2009.

7. Andreou D, Boldt H, Werner M, Hamann C, Pink D and Tunn PU: Sentinel node biopsy in soft tissue sarcoma subtypes with a high propensity for regional lymphatic spreadresults of a large prospective trial. Ann Oncol 24: 1400-1405, 2013.

8. Mavrogenis A, Bianchi G, Stavropoulos N, Papagelopoulos P and Ruggieri P: Clinicopathological features, diagnosis and treatment of clear cell sarcoma/melanoma of soft parts. Hippokratia 17: 298-302, 2013.

9. Egawa Y, Saigo C, Kito Y, Moriki T and Takeuchi T: Therapeutic potential of CPI-613 for targeting tumorous mitochondrial energy metabolism and inhibiting autophagy in clear cell sarcoma. PLoS One 13: e0198940, 2018.

10. Kawashima K, Saigo C, Kito Y, Hanamatsu Y, Egawa Y and Takeuchi T: CD151 confers metastatic potential to clear cell sarcoma of the soft tissue in animal model. Oncol Lett 17: 4811-4818, 2019.

11. MacDougall JR, Croy BA, Chapeau C and Clark DA: Demonstration of a splenic cytotoxic effector cell in mice of genotype SCID/SCID.BG/BG. Cell Immunol 130: 106-117, 1990.

12. Budzynski W and Radzikowski C: Cytotoxic cells in immunodeficient athymic mice. Immunopharmacol Immunotoxicol 16: 319-346, 1994.

13. Sonobe H, Furihata M, Iwata J, Ohtsuki Y, Mizobuchi H, Yamamoto $\mathrm{H}$ and Kumano O: Establishment and characterization of a new human clear-cell sarcoma cell-line, HS-MM. J Pathol 169: 317-322, 1993. 
14. Sonobe H, Takeuchi T, Taguchi T, Shimizu K, Iwata J, Furihata M and Ohtsuki Y: Further characterization of the human clear cell sarcoma cell line HS-MM demonstrating a specific $\mathrm{t}(12 ; 22)$ (q13;q12) translocation and hybrid EWSR1/ATF-1 transcript. J Pahol 187: 594-597, 1999.

15. Takeuchi T, Adachi Y and Nagayama T: A WWOX-binding molecule, transmembrane protein 207 , is related to the invasiveness of gastric signet-ring cell carcinoma. Carcinogenesis 33 548-554, 2012.

16. Asano Y, Takeuchi T, Okubo H, Saigo C, Kito Y, Iwata Y, Futamura $M$ and Yoshida K: Nuclear localization of LDL receptor-related protein $1 \mathrm{~B}$ in mammary gland carcinogenesis. J Mol Med (Berl) 97: 257-268, 2019.

17. Livak KJ and Schmittgen TD: Analysis of relative gene expression data using real-time quantitative PCR and the 2(-Delta C(T)) method. Methods 25: 402-408, 2001.

18. Kanda Y: Investigation of the freely available easy-to-use software 'EZR' for medical statistics. Bone Marrow Transplant 48 : 452-458, 2013

19. R Core Team: R: A language and environment for statistical computing. R Foundation for Statistical Computing, Vienna, 2014.

20. Chan CJ, Martinet L, Gilfillan S, Souza-Fonseca-Guimaraes F, Chow MT, Town L, Ritchie DS, Colonna M, Andrews DM and Smyth MJ: The receptors CD96 and CD226 oppose each other in the regulation of natural killer cell functions. Nat Immunol 15 431-438, 2014
21. Ochoa MC, Minute L, Rodriguez I, Garasa S, Perez-Ruiz E, Inogés S, Melero I and Berraondo P: Antibody-dependent cell cytotoxicity: Immunotherapy strategies enhancing effector NK cells. Immunol Cell Biol 95: 347-355, 2017.

22. Faltas B: Cornering metastases: Therapeutic targeting of circulating tumor cells and stem cells. Front Oncol 2: 68, 2012.

23. Narasimhan PB, Eggert T, Zhu YP, Marcovecchio P, Meyer MA, Wu R and Hedrick CC: Patrolling monocytes control NK cell expression of activating and stimulatory receptors to curtail lung metastases. J Immunol 204: 192-198, 2020.

24. Bi J and Tian Z: NK cell dysfunction and checkpoint immunotherapy. Front Immunol 10: 1999, 2019.

25. López-Soto A, Gonzalez S, Smyth MJ and Galluzzi L: Control of metastasis by NK cells. Cancer Cell 32: 135-154, 2017.

(i) $\mathrm{S}$ This work is licensed under a Creative Common Attribution-NonCommercial-NoDerivatives 4.0 International (CC BY-NC-ND 4.0) License. 